\title{
Aproximación a un Modelo de Bienestar en Adultos Ecuatorianos
}

\author{
Approach to a Wellbeing Model in Ecuadorian Adults
}

\author{
Sandra Lima-Castro ${ }^{1}$, Eva Peña-Contreras ${ }^{2}$, Mónica Aguilar-Sizer ${ }^{3}$, Alexandra Bueno Pacheco ${ }^{4}$ y \\ Paúl Arias-Medina ${ }^{5}$
}

\begin{abstract}
Resumen
El objetivo del estudio fue proponer y evaluar un modelo estructural que explique el bienestar como sinónimo de salud mental positiva de los ecuatorianos a partir de un conjunto de variables psicosociales asociadas evaluadas a través de cuestionarios. La muestra incluyó 1064 adultos de la ciudad de Cuenca- Ecuador. Los resultados se analizaron con los softwares SPSS 20.0 y AMOS 23.0, empleando el método de mínimos cuadrados generalizados. Se probaron modelos parciales, el modelo estructural de ajuste más satisfactorio obtuvo los siguientes resultados: $\mathrm{GFI}=.91$; $\mathrm{AGFI}=.90$; RMR=.205; RMSEA=.041; CFI=.90. Se incluyen en su estructura tres factores (Propósito, Relaciones Positivas y Crecimiento), además la dimensión de bienestar social. La autovaloración de salud, la autoeficacia y la gratitud son predictores importantes de la salud mental mientras que el incremento de las emociones negativas tiene un efecto negativo.
\end{abstract}

Palabras clave: bienestar, salud mental positiva, autoeficacia, gratitud

\begin{abstract}
The aim of this study was to propose and evaluate a structural model that explains well-being as a synonym of positive mental health in Ecuadorians, taking into consideration a group of associated psychosocial variables evaluated through questionnaires. The sample included 1064 adults, inhabitants of CuencaEcuador. The results were analysed with the software SPSS 20.0 and AMOS 23.0, using the generalized least squares method. Partial models were tested, the most satisfactory structural adjustment model obtained the following results; GFI=.91; AGFI=.90; RMR=.205; RMSEA=.041; $\mathrm{CFI}=.90$. Three factors are included in the structure (Purpose, Positive Relationships and Growth), as well as the social well-being dimension. Selfassessment of health, self-efficacy and gratitude are important predictors of mental health while the increase of negative emotions showed a negative effect.
\end{abstract}

Keywords: well-being, positive mental health, self-efficacy, gratitude

El presente estudio es parte de un proyecto más amplio denominado "Variables asociadas al Bienestar de Personas con y sin Discapacidad", proyecto ganador del XIII concurso con apoyo y financiamiento del Departamento de Investigación de la Universidad de Cuenca bajo la dirección del PhD. Mauricio Espinoza.

\footnotetext{
${ }^{1}$ Psicóloga Clínica. Docente Investigadora, Universidad de Cuenca, Ecuador. Av. 12 de abril y Avenida Loja, 010102 . Tel.: +593 985739491. Correo: sandra.lima@ucuenca.edu.ec

${ }^{2}$ Psicóloga Clínica. Docente Investigadora, Universidad de Cuenca, Ecuador. Av. 12 de abril y Avenida Loja, 010102, Tel .: +593 998494242. Correo: eva.pena@ucuenca.edu.ec

${ }^{3}$ Psicóloga Clínica. Docente Investigadora, Universidad de Cuenca, Ecuador. Av. 12 de abril y Avenida Loja, 010102 , Tel .: +593 998420911. Correo: monica.aguilars@ucuenca.edu.ec

${ }^{4}$ Psicóloga Clínica, Docente- Investigadora, Universidad del Azuay, Ecuador. Av. 24 de mayo 7-77, 010107, Tel .: +593 991506998. Correo: abueno@uazuay.edu.ec

${ }^{5}$ Economista, Docente Investigador, Universidad de Cuenca, Ecuador. Av. 12 de abril y Avenida Loja, 10102, Tel .: +593 999287616. Correo: paul.arias@ucuenca.edu.ec
}

Revista Iberoamericana de Diagnóstico y Evaluación - e Avaliação Psicológica. RIDEP · No51 V Vol.2 · 5-18 2019

ISSN: 1135-3848 print /2183-6051online 


\section{Introducción}

El desarrollo de modelos conceptuales multidimensionales para el estudio del bienestar como sinónimo de salud mental positiva ha generado gran interés científico pues se espera que sirvan como fundamento para el abordaje de la salud mental desde un enfoque centrado en la promoción de la salud (Huppert \& So, 2013).

El modelo de la Organización Mundial de la Salud, define a la salud mental como "un estado de bienestar en el cual el individuo es consciente de sus propias capacidades, afronta las tensiones normales de la vida, puede trabajar de forma productiva y fructífera y es capaz de hacer una contribución a su comunidad" (World Health Organization [WHO], 2004, p2). Esta visión positiva de la salud mental coincide con la propuesta moderna de la psicología positiva, que sugiere que para obtener un mejor entendimiento de la complejidad del bienestar humano o salud mental positiva se requiere el desarrollo de modelos multidimensionales del bienestar. Por un lado estos modelos integran teorías y componentes de la perspectiva hedónica que estudia el bienestar subjetivo y emocional, es decir la experiencia de felicidad y satisfacción con la vida y, por otro lado, de la perspectiva eudaimónica que estudia el bienestar psicológico y social, o el funcionamiento humano óptimo (Huppert 2009; Huppert \& So, 2013; Keyes, 2005; Seligman, 2011; Wong, 2011). El potencial de integrar teorías y componentes tanto de la perspectiva hedónica como la eudaimónica, permite la conceptualización de la salud mental positiva como un constructo multidimensional que refleja el bienestar general compuesto por factores del bienestar subjetivo, emocional, psicológico y social (Keyes, 2005). Por lo tanto, la salud mental positiva (SMP) puede considerarse como la experiencia de sentirse bien y funcionar efectivamente a nivel intrapersonal e interpersonal al afrontar los desafíos de la vida (Huppert \& So, 2013; Keyes, 2005). Al revisar la literatura relacionada con Psicología Positiva, encontramos que los constructos bienestar y florecimiento se utilizan como sinónimos de salud mental positiva, así los concebimos en este estudio.

Inicialmente, Keyes $(2005,2007)$ propuso un modelo que integra los componentes hedónicos y eudaimónicos, para evaluar la salud mental positiva a través de tres dimensiones: bienestar emocional (afecto positivo alto y afecto negativo bajo), bienestar psicológico (auto-aceptación, crecimiento personal, propósito en la vida, dominio del medio ambiente, la autonomía y las relaciones positivas con los demás) y bienestar social (aceptación social, actualización social, la contribución social, cohesión social e integración social). Además, la salud mental positiva y la enfermedad mental son dos dimensiones unipolares correlacionadas entre sí, cuestionando el supuesto que tanto salud mental y enfermedad mental son extremos de una única dimensión unipolar. Para comprobar su conceptualización, Keyes (2005) llevó a cabo el primer análisis estructural de salud mental y enfermedad mental latente mostrando que dos factores unipolares diferentes proporcionan la mejor solución.

Diener et al. (2010), optan por un modelo multidimensional del bienestar amplificando su propuesta teórica al integrar los componentes: propósito en la vida, relaciones positivas, compromiso, competencia, autoestima, optimismo y contribución al bienestar de los demás.

Por otro lado, la teoría de Seligman, denominada PERMA, por sus siglas en inglés (Positive Emotions, Engagement, Relationships, Meaning, \& Achievement), también resalta como un modelo multidimensional sobre el bienestar o florecimiento. El autor plantea que el bienestar está compuesto por cinco elementos independientes y medibles que son: emociones positivas, compromiso, relaciones personales, significado, propósito de vida y por último logro; además de estar asociada a fortalezas de carácter (Forgeard, Jayawickreme, Kern, \& Seligman, 2011). Aunque el modelo es relativamente reciente, existen estudios empíricos que lo respaldan (Hone, Jarden, Schofield, \& Duncan, 2014; Kern, Waters, Adler, \& White, 2015).

Huppert y So (2013) proponen un marco conceptual reciente que integra los aspectos hedónicos y eudaimónicos del bienestar. Su enfoque multidimensional integra los componentes: competencia, estabilidad emocional, compromiso, significado, optimismo, emoción positiva, relaciones positivas, resiliencia, autoestima $\mathrm{y}$ vitalidad. 
En definitiva, los modelos multidimensionales van obteniendo cada vez más reconocimiento (Huppert \& So, 2013; Keyes, 2005, 2007; Ryan \& Deci, 2001; Castro-Solano, 2011) pero no existe un acuerdo sobre cuál debe ser utilizado en la investigación o en la política pública (Hone et al., 2014). Además, la mayoría de estudios se han centrado en analizar por separado predictores ya sea del bienestar hedónico o del bienestar eudaimónico, sólo se han publicado algunos estudios sobre predictores de la salud mental positiva y esta evidencia proviene únicamente del contexto anglosajón o europeo (Huppert \& So, 2013; Keyes, 2005, 2007; Keyes \& Simoes, 2012). Estos estudios aún son escasos en Latinoamérica (Barrera-Guzmán \& Flores-Galaz, 2015; Reyes-Jarquín \& Hernández-Pozo, 2012) e inexistentes en el Ecuador. A continuación, desarrollamos un marco teórico de referencia para nuestro estudio en el cual describimos algunas variables que se relacionan transculturalmente indistintamente con el bienestar hedónico o eudaimónico.

\section{Relación entre la autovaloración de salud y bienestar}

La autovaloración de salud hace referencia a la sensación general de bienestar físico de un individuo (George \& Bearon, 1980). Esta autovaloración en algunas ocasiones no coincide con el punto de vista médico, sin embargo, ha demostrado ser un buen predictor de mortalidad (Idler \& Benyamini, 1997; Lucas, Dyrenforth, \& Diener, 2008), estar asociada con niveles más altos de salud mental positiva (Van Lente et al, 2012), y está altamente correlacionada con el bienestar subjetivo (Schneider et al., 2004; KöötsAusmees \& Realo, 2015). Por otro lado, existe evidencia de que la percepción subjetiva de la salud es más alta en los estudiantes más jóvenes y de nivel socioeconómico más alto (Waure, Soffiani, Virdis, Poscia, \& Pietro, 2015). La autovaloración de salud declina con la edad y su asociación con la salud mental es más significativa en muestras de adultos mayores (Pinquart, 2001).

\section{Relación entre emociones negativas y salud mental}

Son consideradas emociones negativas la ansiedad, la depresión, la ira y el miedo. Estos estados afectivos negativos están asociados con enfermedades mentales en influyen en su inicio, desarrollo y mantenimiento (Carrillo, Collado, Rojo, \& Staats, 2006; Keyes, 2007), así como con un bienestar subjetivo deficiente tanto en países industrializados como en países en vías de desarrollo (Pressman, Gallagher, \& Lopez, 2013). Consedine y Moskowitz (2007) afirman que, con la excepción de la ansiedad y la depresión, existe poca literatura que ha estudiado emociones negativas discretas y su relación con la salud; entre estas emociones se incluyen la repulsión, vergüenza, tristeza, culpa, ira y miedo.

Aunque a nivel global las emociones negativas contribuyen a un menor bienestar subjetivo, la magnitud de estos efectos varía considerablemente en distintos contextos ya que las emociones son fenómenos culturales (Consedine, Magai, Cohen, \& Gillespie, 2002; Curhan et al., 2014; Pressman, Gallagher \& Lopez, 2013). Además, las emociones negativas predicen con más intensidad resultados de salud en el corto plazo, en contraste con las emociones positivas que pronostican resultados de salud en el largo plazo (Diener \& Chan, 2011).

\section{Relación entre autoeficacia y bienestar}

La autoeficacia general se define como la confianza en la propia capacidad para manejar diferentes situaciones y exigencias en una variedad de contextos (Bandura 1977; Schwarzer, Bäßler, Kwiatek, Schröder, \& Zhang, 1997). Varios estudios transculturales comprueban que es un constructo unidimensional y universal (Luszczynska, Gutiérrez-Doña, \& Schwarzer, 2005; Löve, Moore, \& Hensing, 2012), que está asociado significativamente con las dimensiones que integran el bienestar psicológico (González Cabanach, Valle Arias, Freire Rodríguez, \& Ferradás Canedo, 2012; Ryan \& Deci, 2001) y la salud mental (Gokbayrak, 2016). También se han encontrado asociaciones negativas altas entre la autoeficacia, depresión y ansiedad en diferentes culturas estudiadas (Luszczynska et al., 2005). Además, la autoeficacia está asociada con mayor probabilidad de evaluar la salud como satisfactoria, estar menos enfermos o depresivos (Olivari-Medina \& Urra-Medina, 2007) y con el mantenimiento de conductas saludables (Luszczynska \& Schwarzer, 2005), mostrando en 
general consecuencias positivas para el individuo (Klein-Heßling, Lohaus, \& Ball, 2005; ReyesJarquín \& Hernández-Pozo, 2012).

\section{Relación entre gratitud y bienestar}

La gratitud puede ser considerada como una emoción que se produce al interpretar la ayuda o apoyo recibido como costosa, valiosa y altruista (Wood, Froh, \& Geraghty, 2008). También puede ser estudiada como un rasgo, es decir, la tendencia de las personas a experimentar gratitud en respuesta a situaciones cotidianas, considerándose una fortaleza psicológica (McCullough, Emmons, \& Tsang, 2004; Peterson \& Seligman, 2004).

Existe suficiente evidencia empírica actual que demuestra que la gratitud se relaciona positivamente con una variedad de formas de bienestar (Alarcón \& Caycho, 2015; Jackson, van de Vijver, \& Fouché, 2014; Lyubomirsky, Dickerhoof, Boehm, \& Sheldon, 2011; Wood et al., 2010). La gratitud estimula cambios positivos en las conductas sociales de las personas, a raíz del efecto de reciprocidad (Dunn \& Schweitzer, 2005; Emmons \& McCullough, 2003, Tsang, 2006). Asimismo, se ha demostrado el efecto de la gratitud en el aumento del sistema inmune (Myers, 1993). La gratitud puede explicar el 20\% de las diferencias individuales con la satisfacción con la vida (Wood, Maltby, Gillett, Linley, \& Joseph, 2008). Sujetos que puntúan alto en la tendencia a experimentar gratitud, obtienen altos valores en empatía, son evaluados por sus pares como más generosos con su tiempo y sus recursos y otorgan poca importancia a los bienes materiales (McCullough, Emmons, Kilpatrick, \& Larson, 2001).

\section{Relación entre bienestar social y salud mental positiva}

El bienestar social es un concepto multidimensional que incluye: la integración social, la aceptación social, la contribución social, actualización social y la cohesión social y está relacionado con salud mental positiva, mayor bienestar subjetivo y psicológico (Diener \& Seligman 2002, 2004; Huppert 2009; Joshanloo, Sirgy, \& Park, 2018; Keyes, 1998; Van Lente et al., 2012). Según Keyes (1998) la contribución social se refiere a la percepción de que se es un miembro vital de la sociedad, que se tiene algo útil que ofrecer al bien común. Los niveles de integración y contribución social son altos en personas implicadas en acciones solidarias, que poseen un sentimiento de pertenencia y lazos sociales fuertemente arraigados.

En definitiva, los factores positivos pueden permitir a los individuos y a las comunidades vivir plenamente y superar aquella visión patogénica de la salud que se centraba únicamente en la presencia de déficits (Gancedo, 2008; Seligman, 2003). Dadas las anteriores consideraciones conceptuales sobre las investigaciones en el área, la presente investigación propone y evalúa un modelo estructural que explique la salud mental centrada en el bienestar a partir del conjunto de variables psicosociales asociadas. Nuestra propuesta de evaluación se encuentra en concordancia con la de Seligman (2003, 2011), Keyes (2005, 2007), y la Organización Mundial de la Salud ya que lejos de diferir la una de la otra, se complementan y concuerdan en que una evaluación integral del bienestar como sinónimo de salud mental positiva, abarca tanto los aspectos hedónicos como eudaimónicos del bienestar. Estos modelos no han sido probados en una muestra ecuatoriana, por lo cual nuestra medida integradora del bienestar incorpora variables asociadas a un funcionamiento positivo y relevantes a nivel transcultural, mismas que hemos señalado en la revisión de la literatura. Por esta razón se considerará al bienestar como elemento central y se evaluará su relación con la gratitud, la autoeficacia, la salud autopercibida, el bienestar social y las emociones negativas.

\section{Métodos}

El estudio se desarrolló bajo un enfoque cuantitativo de tipo transversal con alcance descriptivo y correlacional. Se analizó las relaciones entre variables observadas y latentes, haciendo uso de Modelos de Ecuaciones Estructurales (SEM), los cuales proporcionan un marco general para el análisis estadístico de las relaciones entre distintas variables (Bollen, 1989; Hayduk, 1987).

\section{Participantes}

En el estudio participaron 1,064 personas de las cuales 387 son hombres (36.4\%) y 677 
mujeres $(63.6 \%)$ en edades comprendidas entre 18 y 89 años con una media de 48.19 años y una DT de 21.566. Del total de la muestra se estudiaron a 445 personas en la adultez temprana (41.8\%), 248 personas en la adultez media (23.3\%) y 371 personas de la adultez tardía (34.9\%). Respecto al nivel de estudios 44 (4.1\%) personas reportan no tener instrucción formal, 169 (15.9\%) no tienen estudios de bachillerato, 297 (27.9\%) con estudios de bachillerato, $554(52.1 \%)$ con estudios universitarios. Además, 175 (16.4\%) refirieron padecer de algún tipo de discapacidad. La muestra es de tipo probabilístico calculado con un porcentaje de error de $3.21 \%$ y un nivel de confianza de $97 \%$. Los participantes del estudio fueron contactados en instituciones laborales y centros públicos y privados de atención en general de la ciudad de Cuenca desde enero del 2016 hasta enero del 2017 con alta concurrencia de transeúntes. La participación de los sujetos fue voluntaria y anónima, Una vez que se obtuvo el consentimiento informado en la cual se explicaban los objetivos de la investigación se procedió a aplicar una encuesta sobre datos sociodemográficos y la aplicación de las escalas que tuvo una duración aproximada de 25 minutos. Cada participante respondió a los cuestionarios en una sola sesión.

\section{Instrumentos}

Cuestionario de características sociodemográficas de los participantes. Se elaboró un cuestionario ad hoc para caracterizar la muestra en función de características sociodemográfica: (sexo, edad, estado civil, empleo, nivel de estudios, ingresos económicos y discapacidad).

Perfil PERMA. Se utilizó una adaptación al español de la escala del Perfil PERMA para su uso en el contexto ecuatoriano (Lima-Castro, Peña-Contreras, Cedillo-Quizhpe, \& CabreraVélez, 2017) basado en la versión original de PERMA- Profiler (Butler \& Kern, 2016). La versión adaptada está compuesta por 15 ítems, que evalúan los cinco pilares del bienestar: emociones positivas, compromiso, relaciones, significado y logros, asignándose 3 ítems para cada factor. Cada ítem se califica en una escala Likert de 11 puntos, que va de 0 (nunca) a 10 (siempre) o 0 (nada) a 10 (completamente).
Escala de Autoeficacia general. Para evaluar la autoeficacia se utilizó la General Self-Efficacy Scale, en su versión de adaptación para población ecuatoriana de Bueno-Pacheco, Lima-Castro, Peña-Contreras, Cedillo-Quizhpe, \& AguilarSizer (2017). Es una escala de 10 ítems auto administrada diseñada para evaluar el sentimiento de competencia personal para afrontar distintas situaciones vitales difíciles. Se evalúa cada ítem en función del grado en que el sujeto cree que esa afirmación sobre su capacidad para resolver problemas es cierta, en una escala Likert de 4 puntos que va de 1 (nunca) a 4 (siempre).

Escala de Gratitud. Adaptación al español de la escala de Gratitud disposicional para su uso en el contexto ecuatoriano GQ-6 (Cabrera-Vélez et al., 2017). En su versión adaptada para población ecuatoriana, la escala está integrada por 5 ítems planteados de manera positiva y negativa, que se responden en una escala tipo Likert que va desde el $1=$ Totalmente en desacuerdo, hasta el 7=Totalmente de acuerdo.

Escala de Bienestar Social de Keyes. Es un cuestionario de auto reporte de 25 ítems elaborado para medir el Bienestar Social de Keyes adaptada por Blanco y Díaz (2005). Dicha escala evalúa la percepción que las personas tienen sobre cinco aspectos: Integración Social, Aceptación social, Contribución Social, Actualización Social y Coherencia Social. Para fines de este estudio se utilizaron únicamente dos ítems uno que evalúan la contribución social: Pienso que lo que hago es importante para la sociedad y otro que evalúa la integración social: Siento que soy una parte importante de mi comunidad.

Autovaloración de salud. El perfil PERMA además del bienestar evalúa tres factores independientes que son: salud, emociones negativas y soledad. Para evaluar la autovaloración de salud se usó la escala de la salud basado en la versión original de PERMAProfiler (Butler \& Kern, 2016) que consta 3 ítems pero con algunas modificaciones para su uso en el Ecuador. Los participantes respondieron a los siguientes ítems utilizando un formato de respuesta de categorías ordenadas con puntuaciones comprendidas entre 0 (Para nada) y 10 (completamente). Los ítems usados en esta escala son: 1. ¿En comparación con otras personas de su misma edad y sexo considera que 
tiene un excelente estado de salud?; 2. ¿Qué tan satisfecho con su salud física se siente actualmente?; 3. En general. ¿tiene una excelente salud física?

Emociones Negativas. Otra escala asociada al PERMA es la de emociones negativas Los participantes respondieron a los siguientes ítems utilizando un formato de respuesta de categorías ordenadas con puntuaciones comprendidas entre 0 (Nunca) y 10 (Siempre). Los ítems usados son: 1 . En general. ¿Con qué frecuencia se siente angustiado?; 2. En general. ¿Con qué frecuencia se siente enojado? y 3. En general ¿Con qué frecuencia se siente triste?

\section{Análisis de datos}

\section{Fase exploratoria}

Los análisis estadísticos se realizaron con los softwares SPSS 20.0 y AMOS 23.0. Antes de realizar la estimación del modelo estructural, se efectuó un análisis factorial exploratorio para analizar las distribuciones de los datos obtenidos y seleccionar los métodos de estimación y ajuste adecuados. Previamente, en una muestra piloto de 40 participantes se calculó el coeficiente $\alpha$ de Cronbach para verificar la confiabilidad de cada una de las subescalas utilizadas (Clark \& Watson, 1995). Para cada variable se evaluó la validez de constructo, analizando que su estructural factorial fuese la adecuada y que el porcentaje de varianza explicado fuese de al menos el 50\%. En la tabla 1 se presentan los resultados de la verificación de la consistencia interna de cada una de las escalas utilizadas. Todos los valores del coeficiente $\alpha$ de Cronbach fueron adecuados.

Tabla 1. Verificación de la consistencia interna de las escalas empleadas en el estudio

\begin{tabular}{lc}
\hline Escala & $\begin{array}{c}\text { Coeficiente } \alpha \text { de } \\
\text { Cronbach }\end{array}$ \\
\hline PERMA (Global) & .889 \\
Sub escala Crecimiento & .747 \\
Sub escala Propósito & .818 \\
Sub escala Relaciones & .811 \\
Autoeficacia & .863 \\
Gratitud & .847 \\
Bienestar Social & .773 \\
Autovaloración de Salud & .844 \\
Emociones Negativas & .811 \\
\hline
\end{tabular}

\section{Análisis Confirmatorio}

Para identificar las relaciones entre variables de interés se probó parcialmente cuatro modelos parciales mediante la metodología planteada por los modelos de ecuaciones estructurales. Además, previamente se consideró un modelo alternativo para dimensiones latentes que podrían influir en la medida de bienestar general. Análisis previos indican que en lugar de emplear las cinco dimensiones de la estructura factorial planteada originalmente por Butler y Kern (2016) para el perfil PERMA, se ajusta mejor al contexto el modelo de tres factores por lo que se optó por este modelo debido a que demostró mejor ajuste (Lima-Castro et al., 2017). A partir de este constructo se planteó el modelo original, considerando al Bienestar como el constructo principal, y como variables exógenas que lo afectan: La gratitud, la Autoeficacia y las Emociones Negativas. Por otra parte, las variables endógenas dentro del modelo son: Propósito, Relaciones Positivas, crecimiento, Salud Auto percibida y Bienestar Social (Ver Figura 1).

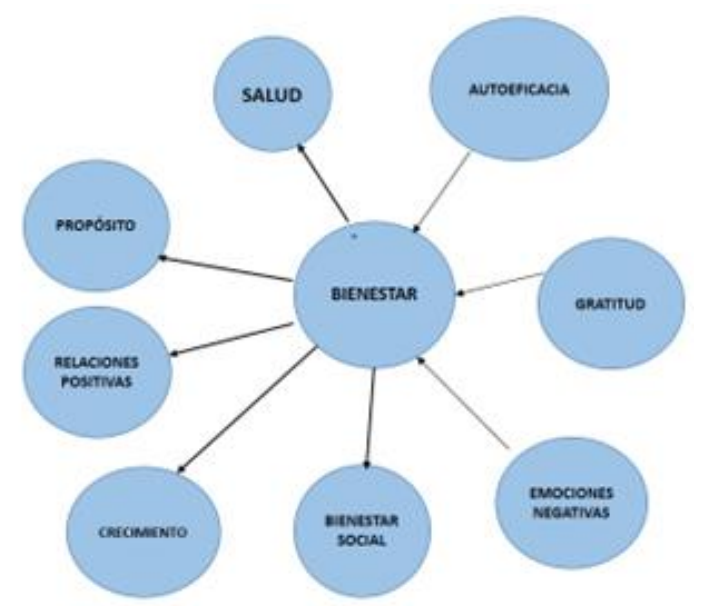

Figura 1. Modelo Inicial de Bienestar General

Para probar la adecuación de los modelos parciales se utilizaron índices de ajuste incremental y absoluto seleccionados según recomendaciones y convenciones de expertos (Browne \& Cudeck, 1993; Hair, Anderson, Tatham, \& Black, 1998; Jöreskog \& Sörbom, 1989). Se seleccionaron el índice RMR (Root Mean Residual) que minimiza el sesgo producido por el tamaño de muestra; índice RMSEA (Root Mean Square Error of Approximation) mide la bondad de ajuste entre el modelo y los datos poblacionales. En ambos casos se considera que 
valores iguales o inferiores a .08 indican un buen ajuste; el índice GFI (Goodness of Fit Index) que representa el grado de ajuste del conjunto del modelo sin tener en cuenta los grados de libertad; AGFI (Adjusted Goodness of Fit Index); índice CFI (Comparative Fit Index) que mide el porcentaje de pérdida que se produce al cambiar el modelo predicho nulo en el ajuste; índice IFI (Incremental Fit Index) que además considera los grados de libertad del modelo; índice TLI (Tucker-Lewis index) que es uno de los índices menos afectados por el tamaño de la muestra. En los últimos cinco índices se considera que valores superiores a .90 indican un buen ajuste.

\section{Resultados}

\section{Análisis Bivariado}

En la Tabla 2 se reportan las correlaciones entre los distintos constructos que constituyen el modelo, las mimas que son significativas, moderadas y positivas entre el bienestar general y la autopercepción de salud, el bienestar social, la autoeficacia y la gratitud que tienen las personas $(\mathrm{r}=.489 ; \mathrm{r}=.642 ; \mathrm{r}=.475$ y $\mathrm{r}=.538, p<.01)$, estos valores indican que todos los constructos convergen hacia una medida común. Por otra parte, todas las variables latentes (Bienestar General, Salud Física, Bienestar Social, Autoeficacia y Gratitud) evidenciaron correlaciones significativas, bajas y negativas con respecto a las emociones negativas de las personas $(\mathrm{r}=-.242 ; \quad \mathrm{r}=-.198 ; \quad \mathrm{r}=-.205 \quad \mathrm{r}=-.234 \mathrm{y} \quad \mathrm{r}=-.149$, $p<.01)$.

\section{Evaluación de Modelos}

Como consecuencia del número amplio de variables en el modelo, se decidió ir ajustando modelos parciales, específicamente se consideraron cuatro modelos y se evaluó el ajuste individual por subescalas, posteriormente se fueron añadiendo otras subescalas y se valoró nuevamente el ajuste en el modelo integrado. Siguiendo este procedimiento, se partió con la escala de Autoeficacia valorando el ajuste y añadiendo otras variables hasta incorporar las relacionadas con el bienestar y obtener así un ajuste del modelo completo. El uso de esta estrategia permitió detectar potenciales casos y variables que afectan el ajuste del modelo facilitando además una evaluación progresiva de la significancia del modelo hasta incorporar todas las variables. En consecuencia, el modelo I sólo considera las variables: Autoeficacia, Gratitud y emociones negativas; el modelo II: Gratitud, Autovaloración de Salud y Emociones negativas; el modelo III incluye las variables: Autoeficacia, Autovaloración de Salud y Emociones Negativas. Finalmente se evalúa el Modelo Completo (identificado como IV) que incluye las variables: Salud Mental Positiva, Bienestar Social, Autovaloración de Salud, Gratitud, Autoeficacia y Emociones Negativas.

La Tabla 3 muestra cada uno de estos modelos y sus respectivos índices de ajuste. Se observa que a medida que el modelo se va completando mejora considerablemente su ajuste global $\left(\chi^{2} / \mathrm{gl}\right)$ aproximándose a 3 y el RMSEA alcanza valores menores a .05 .

Tabla 2. Correlaciones entre la escala de Bienestar, Salud Autopercibida, Contribución Social, Emociones Negativas, Autoeficacia y Gratitud

\begin{tabular}{|c|c|c|c|c|c|c|}
\hline & Bienestar & Salud Física & $\begin{array}{c}\text { Contribución } \\
\text { Social }\end{array}$ & $\begin{array}{c}\text { Emociones } \\
\text { Negativas }\end{array}$ & Autoeficacia & Gratitud \\
\hline Bienestar General & 1 & $.489^{* *}$ & $.642^{* *}$ & $-.242^{* * *}$ & $.475^{* *}$ & $.538^{* *}$ \\
\hline $\begin{array}{l}\text { Autovaloración } \\
\text { Salud }\end{array}$ & & 1 & $.396^{* *}$ & $-.198^{* *}$ & $.232^{* *}$ & $.318^{* * *}$ \\
\hline Bienestar Social & & & 1 & $-.205^{* *}$ & $.414^{* *}$ & $.374^{* *}$ \\
\hline $\begin{array}{l}\text { Emociones } \\
\text { Negativas }\end{array}$ & & & & 1 & $-.234^{* *}$ & $-.149 * *$ \\
\hline Autoeficacia & & & & & 1 & $.293^{* *}$ \\
\hline Gratitud & & & & & & 1 \\
\hline
\end{tabular}

** La correlación es significativa al nivel .01 (bilateral). 
Tabla 3. Comparación de estadísticos de bondad de ajuste de los modelos de predicción de bienestar (salud

\begin{tabular}{|c|c|c|c|c|c|c|c|c|}
\hline \multirow{2}{*}{ Modelo } & \multirow{2}{*}{$\begin{array}{l}\text { Chi-cuadrado } \\
\qquad\left(\chi^{2}\right)\end{array}$} & \multirow{2}{*}{$\begin{array}{c}\text { Grados de } \\
\text { libertad } \\
\text { (gl) }\end{array}$} & \multirow[b]{2}{*}{$\chi^{2} / \mathrm{gl}$} & \multicolumn{5}{|c|}{ Índices de Ajuste $(*)$} \\
\hline & & & & GFI & AGFI & RMR & RMSEA & CFI \\
\hline $\begin{array}{l}\text { I. Autoeficacia + Gratitud y } \\
\text { emociones negativas }\end{array}$ & 1982.61 & 488 & 4.06 & .897 & .881 & .176 & 0.054 & .90 \\
\hline II. Gratitud+ Autovaloración d & & & & & & & & \\
\hline Salud + Emociones negativas & 1248.71 & 291 & 4.29 & .915 & .897 & .159 & 0.056 & .92 \\
\hline $\begin{array}{l}\text { III Autoeficacia + } \\
\text { Autovaloración de Salud + } \\
\text { Emociones Negativas } \\
\text { IV Modelo Completo de }\end{array}$ & 1675.50 & 426 & 3.93 & .905 & .890 & .162 & 0.053 & .91 \\
\hline Salud Mental Positiva & & & & & & & & \\
\hline $\begin{array}{l}\text { Bienestar Social + } \\
\text { Autovaloración de Salud + } \\
\text { Gratitud + Autoeficacia + } \\
\text { Emociones Negativas }\end{array}$ & 1764.64 & 585 & 3.01 & .91 & .90 & .205 & 0.041 & .90 \\
\hline
\end{tabular}

La Tabla 4 muestra los coeficientes estandarizados para las distintas variables latentes que constituyen el modelo de medida. Las cargas estandarizadas $(\beta)$ presentan estadísticos t-Student con valores superiores a \pm 1.96 , lo que indica que son significativamente distintos de cero en la población con un nivel de significación $\alpha=.05$ y los valores de los coeficientes estandarizados oscilan en el intervalo $.37 \leq \beta \leq .88$. La estimación de los errores estandarizados en general es aceptable y varía entre $.06 \leq S E \leq .43$.

En referencia al modelo estructural los valores de los coeficientes estandarizados se muestran en la Tabla 5. Se observa un peso significativo de las variables asociadas al bienestar: Crecimiento y Relaciones positivas $(\beta=.81$ y $\quad \beta=.97$ respectivamente). Como variables exógenas, el Bienestar Social es la variable psicológica que más destaca en la explicación del Bienestar General $(\beta=.76)$, la Salud Autopercibida, tiene una contribución de modera a alta en la percepción del bienestar $(\beta=.59)$. La Gratitud es la variable endógena con un peso moderado en el Bienestar $(\beta=.48)$ y el sentido de Autoeficacia tiene un peso menor dentro del conjunto $(\beta=.28)$.

\section{Discusión y Conclusiones}

La presente investigación demuestra su aporte ya que se basa en planteamientos teóricos sólidos de la Organización Mundial de la Salud contribuyendo a la investigación científica en el contexto Latinoamericano pues propone y evalúa un modelo estructural que explica la salud mental positiva (bienestar general) de los ecuatorianos a partir de un conjunto de variables psicosociales asociadas. Para dar respuesta a este objetivo general en primer lugar se comprobó empíricamente el modelo de la salud mental positiva de Seligman (2011) que señala que la salud mental supone la presencia de cinco componentes: emociones positivas, compromiso, relaciones, significado y logro. Mediante el estudio realizado se pudo corroborar que tres dimensiones latentes demostraron mayor parsimonia para la medida de bienestar en lugar de cinco que es la estructura factorial planteada originalmente por Butler y Kern, (2016). Esta divergencia podría deberse a ciertas características socioculturales de la muestra no analizadas en este estudio. 
Tabla 4. Coeficientes Estandarizados de las variables observadas del modelo de medida

\begin{tabular}{|c|c|c|c|c|}
\hline Relación & & & $\begin{array}{c}\text { Coeficiente } \\
\text { Estandarizado }(\beta)\end{array}$ & $p$ \\
\hline E2 & <--- & PROPOSITO & .73 & .00 \\
\hline M3 & $<---$ & PROPOSITO & .583 & .00 \\
\hline M1 & $<---$ & PROPOSITO & .631 & .00 \\
\hline M2 & $<---$ & PROPOSITO & .775 & .00 \\
\hline A3 & $<---$ & PROPOSITO & .537 & .00 \\
\hline E1 & $<--$ & CRECIMIENTO & 679 & .00 \\
\hline $\mathrm{A} 2$ & $<--$ & CRECIMIENTO & .689 & .00 \\
\hline A1 & $<---$ & CRECIMIENTO & .65 & .00 \\
\hline H1 & $<---$ & SALUD & .89 & .00 \\
\hline $\mathrm{H} 2$ & $<---$ & SALUD & .837 & .00 \\
\hline H3 & $<--$ & SALUD & .627 & .00 \\
\hline N3 & $<---$ & EMO_NEG & .844 & .00 \\
\hline N2 & $<---$ & EMO_NEG & .659 & .00 \\
\hline N1 & $<--$ & EMO_NEG & .652 & .00 \\
\hline AUTO1 & $<--$ & AUTOEF & .374 & .00 \\
\hline AUTO2 & $<--$ & AUTOEF & .392 & .00 \\
\hline AUTO3 & $<--$ & AUTOEF & .378 & .00 \\
\hline AUTO4 & $<---$ & AUTOEF & .685 & .00 \\
\hline AUTO5 & $<---$ & AUTOEF & .75 & .00 \\
\hline AUTO6 & $<---$ & AUTOEF & .6 & .00 \\
\hline AUTO7 & $<--$ & AUTOEF & .686 & .00 \\
\hline AUTO8 & $<--$ & AUTOEF & .601 & .00 \\
\hline AUTO9 & $<--$ & AUTOEF & .615 & .00 \\
\hline AUTO10 & $<---$ & AUTOEF & .593 & .00 \\
\hline G_1 & $<---$ & GRATITUD & .631 & .00 \\
\hline G_2 & $<---$ & GRATITUD & .783 & .00 \\
\hline G_3 & $<---$ & GRATITUD & .791 & .00 \\
\hline G_4 & $<---$ & GRATITUD & .683 & .00 \\
\hline G_5 & $<---$ & GRATITUD & .64 & .00 \\
\hline INT_SOCIAL & $<---$ & B. SOCIAL & .759 & .00 \\
\hline CONTRI_SOC & $<---$ & B. SOCIAL & .77 & .00 \\
\hline P3 & $<---$ & REL_POS & .761 & .00 \\
\hline $\mathrm{P} 2$ & $<--$ & REL_POS & .562 & .00 \\
\hline $\mathrm{P} 1$ & $<---$ & REL_POS & .549 & .00 \\
\hline $\mathrm{R} 3$ & $<---$ & REL_POS & .658 & .00 \\
\hline $\mathrm{R} 2$ & $<---$ & REL_POS & .612 & .00 \\
\hline
\end{tabular}

Nomenclatura ítems PERMA: Emociones Positivas (P1, P2 y P3); Compromiso (E1, E2 y E3); Relaciones (R1, R2 y R3); Significado (M1, M2 y M3);Logro (A1, A2 y A3); Salud (H1,H2 y H3); Emociones Negativas (N1, N2 y N2). Autoeficacia (AUTO).Gratitud (G).

Tabla 5. Modelo Estructural de las variables latentes del modelo estructural

\begin{tabular}{lllcc}
\hline Relación & & & $\begin{array}{c}\text { Coeficiente } \\
\text { Estandarizado }(\beta)\end{array}$ & $p$ \\
\hline BIENESTAR GENERAL & $<---$ & AUTOEFICACIA & .284 & .00 \\
BIENESTAR GENERAL & $<--$ & GRATITUD & .479 & .00 \\
BIENESTAR GENERAL & $<---$ & EMO_NEG & -.149 & .01 \\
CRECIMIENTO & $<---$ & BIENESTAR GENERAL & .813 & .00 \\
B. SOCIAL & $<---$ & BIENESTAR GENERAL & .755 & .00 \\
REL_POS & $<---$ & BIENESTAR GENERAL & .974 & .00 \\
SALUD & $<---$ & BIENESTAR GENERAL & .587 & .00 \\
\hline
\end{tabular}




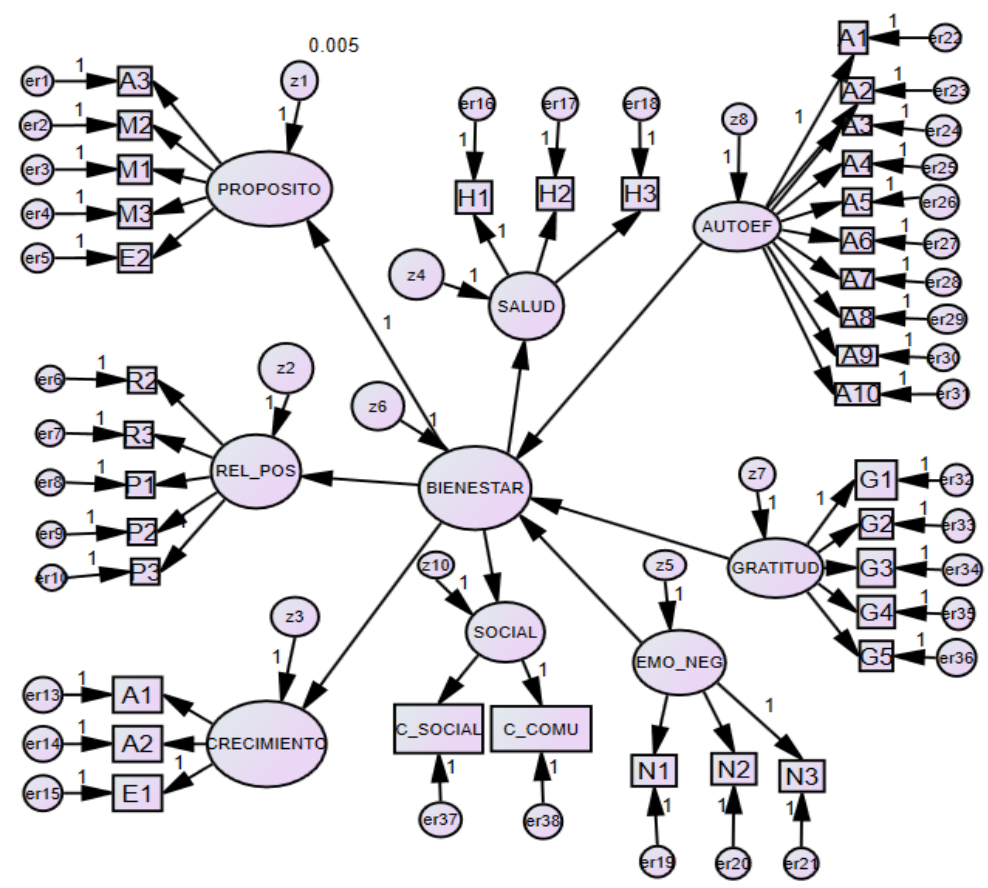

Figura 2. Modelo Final de Salud mental positiva (Bienestar General). Chi cuadrado =1764.64.12; gl=585; RMSEA=.04; GFI=.90; AGFI=.91

Con el modelo estructural completo propuesto se considera que la autoeficacia, gratitud, emociones negativas y salud son variables predictoras del bienestar. En primera instancia se pudo confirmar que el modelo de Bienestar de tres factores presentado por Lima-Castro et al. (2017) presenta una excelente estructura para evaluar el bienestar a partir de las siguientes dimensiones: propósito, relaciones socio-emocionales y crecimiento, aportando significativamente a la medición del Bienestar en los ecuatorianos. Por otra parte, vemos que el Bienestar social, aparece como una dimensión significativa del bienestar general comprobando empíricamente la propuesta teórica de Keyes $(2005,2007)$ y coincidiendo con otros estudios que señalan que el bienestar social está asociado con salud mental positiva y negativa (Van Lente et al., 2012).

Además, se evidenció que tanto la autoeficacia como la tendencia a experimentar gratitud son variables relacionadas de manera positiva. Aunque para este par de variables se consideró su efecto sobre el bienestar, vemos que este se torna significativo y coincide con la investigación existente tanto para el constructo autoeficacia (González Cabanach et al., 2012; Gokbayrak, 2016; Ryan \& Deci, 2001) como para la tendencia a experimentar gratitud (Lyubomirsky et al.,
2011, Alarcón \& Caycho, 2015, Wood et al., 2010). Al igual que en otros estudios la presente investigación demuestran la relación entre salud física y bienestar (Diener \& Chan, 2011). La autovaloración de la salud física tiene un efecto importante y significativo sobre la salud mental positiva al ser una fuente de información importante utilizada para construir la valoración global del bienestar.

Como se ha mencionado anteriormente, el campo de la piscología positiva ha concentrado sus esfuerzos en el estudio de las fortalezas y potencialidades humanas. Park, Peterson, \& Seligman, (2004) definen las fortalezas de carácter como aquellos rasgos positivos reflejados en pensamientos, sentimientos y comportamientos llegando a identificarse 24 fortalezas de carácter agrupadas en 6 categorías; sin embargo, solo algunas fortalezas de carácter entre ellas la gratitud parecen tener una relación directa con el bienestar subjetivo (Brdar \& Kashdan, 2010). Lo hallazgos de nuestro estudio coinciden con otros que señalan que la gratitud se relaciona positivamente con una variedad de formas de bienestar (Alarcón \& Caycho, 2015; Lyubomirsky, Dickerhoof, Boehm, \& Sheldon, 2011; Wood et al., 2010) Además podemos destacar que a nivel latinoamericano la gratitud 
inclusive es una fortaleza que tiene manifestaciones culturales a través de canciones, poemas, etc. Por lo que sería necesario seguir profundizando su estudio como recurso protector ante determinados problemas psicopatológicos

Un incremento de las emociones negativas afecta sustancialmente el bienestar general de las personas y en un menor grado, su sentido de autoeficacia, gratitud, autovaloración de salud y bienestar social. Estos resultados coinciden con estudios que señalan que la salud mental aumenta al maximizar los sentimientos positivos $y$ agradables, al tiempo que minimiza los sentimientos negativos y desagradables (Diener \& Suh 1997, Diener et al., 1999; Keyes, 2007)

El estudio realizado cuenta con limitaciones debido al carácter transversal lo que hace difícil establecer una causalidad. Es recomendable analizar el ajuste del modelo planteado en diferentes poblaciones clasificadas por edad, sexo, región, o culturalmente distintas, etc. Sin embargo, estos hallazgos motivan la importancia de conocer y estudiar variables psicosociales asociadas a la salud mental puesto que generar información permitiría diseñar intervenciones novedosas desde una perspectiva integral de promoción para la salud mental.

\section{Referencias}

Alarcón, R., \& Caycho Rodríguez, T. (2015). Relaciones entre gratitud y felicidad en estudiantes universitarios de Lima metropolitana. Psychologia. Avances de la disciplina, 9, 59-69.

Bandura, A. (1977). Self-efficacy: Toward a unifying theory of behavioral change. Psychological Review, 84, 191-215. doi:1.1037/0033-295X.84.2.191

Barrera-Guzmán, M. L., \& Flores-Galaz, M. M. (2015). Construcción de una Escala de Salud Mental Positiva para adultos en población mexicana. Revista Iberoamericana de Diagnóstico y Evaluación Psicológica - e Avaliação Psicológica, 39, 22-33.

Blanco, A., \& Díaz, D. (2005). El bienestar social: Su concepto y medición. Psicothema, 17(4), 582-589. Recuperado de https://goo.gl/PeY1tI
Bollen, K. A. (1989). Structural equations with latent variables. New York, NY: John Wiley \& Sons.

Brdar, I., \& Kashdan, T. B. (2010). Character strengths and well-being in Croatia: An empirical investigation of structure and correlates. Journal of Research in Personality, 44(1), 151-154. https://doi.org/10.1016/j.jrp.2009.12.001

Browne, M. W., \& Cudeck, R. (1993). Alternative ways of assessing model fit. Sage Focus Editions, 154, 136-136. Recuperado de https://goo.gl/5abflx

Bueno-Pacheco, A., Lima-Castro, S., PeñaContreras, E., Cedillo-Quizhpe, C., \& Aguilar-Sizer, M. (2017). Adaptación al español de la escala de autoeficacia general para su uso en el contexto ecuatoriano. En imprenta. Facultad de Psicología, Universidad de Cuenca. Ecuador

Butler, J., \& Kern, M. L. (2016). The PERMAProfiler: A brief multidimensional measure of flourishing. International Journal of Wellbeing, $\quad 6(3), \quad 1-48$. doi:10.5502/ijw.v6i3.526

Cabrera-Vélez, M., Lima-Castro, S., PeñaContreras, E., Aguilar-Sizer, M., BuenoPacheco, A., \& Arias-Media, P. (2017). Adaptación del Cuestionario de Gratitud (CG-6) en una muestra de Ecuatoriana. Manuscrito no publicado. Facultad de Psicología, Universidad de Cuenca. Ecuador.

Carrillo, J. M., Collado, S., Rojo, N., \& Staats, A. W. (2006). El papel de las emociones positivas y negativas en la predicción de depresión: El principio de adición de las emociones en el conductismo psicológico. Clínica y Salud, 17(3), 277-295. Recuperado de https://goo.gl/4GH4pt

Castro-Solano, A. (2011). Las rutas de acceso al bienestar. Relaciones entre bienestar hedónico y eudamónico. Un estudio en población argentina. Revista Iberoamericana de Diagnóstico y Evaluación Psicológica - e Avaliação Psicológica, 31, 37-57.

Clark, L. A., \& Watson, D. (1995). Constructing validity: Basic issues in objective scale development. Psychological Assessment, 7(3), 309. doi:1.1037/1040-359.7.3.309

Consedine, N. S., Magai, C., Cohen, C. I., \& Gillespie, M. (2002). Ethnic variation in the 
impact of negative affect and emotion inhibition on the health of older adults. The Journals of Gerontology. Series B, Psychological Sciences and Social Sciences, 57(5), 396-408. doi:10.1093/geronb/57.5.P396

Consedine, N. S., \& Moskowitz, J. T. (2007). The role of discrete emotions in health outcomes: A critical review. Applied and Preventive Psychology, 12(2), 59-75.

Diener, E. D., \& Suh, E. (1997). Measuring quality of life: Economic, social, and subjective indicators. Social Indicators Research, $\quad 40, \quad 189-216$. doi:10.1023/A:1006859511756

Diener, E., Suh, E. M., Lucas, R. E., \& Smith, H. L. (1999). Subjective well-being: Three decades of progress. Psychological Bulletin, 125(2), 276-302. doi:10.1037/0033-2909.125.2.276

Diener, E., \& Seligman, M. E. P. (2002). Very happy people. Psychological Science, 13, 8184

Diener, E., \& Seligman, M. E. P. (2004). Beyond money: Toward an economy of well-being. Psychological Science in the Public Interest, 5,1-30.

Diener, E., Wirtz, D., Tov, W., Kim-Prieto, C., Choi, D. W., Oishi, S., \& Biswas-Diener, R. (2010). New well-being measures: Short scales to assess flourishing and positive and negative feelings. Social Indicators Research, 97(2), 143-156. doi:1.1007/s11205-009-9493-y

Diener, E., \& Chan, M. Y. (2011). Happy people live longer: Subjective well-being contributes to health and longevity. Applied Psychology: Health and Well-Being, 3(1), 1-43. doi:10.1111/j.1758-0854.2010.01045.x

Dunn, J., \& Schweitzer, M. E. (2005). Why good employees make unethical decisions: The role of reward systems, organizational culture, and managerial oversight. Managing organizational deviance, 39-68.

Emmons, R. A., \& McCullough, M. E. (2003). Counting blessings versus burdens: An experimental investigation of gratitude and subjective well-being in daily life. Journal of Personality and Social Psychology,84(2), 377. doi:1.1037/0022-3514.84.2.377

Forgeard, M. J. C., Jayawickreme, E., Kern, M. L., \& Seligman, M. E. P. (2011). Doing the right thing: Measuring well-being for public policy. International Journal of Wellbeing, 1(1). doi:10.5502/ijw.v1i1.15

Gancedo, M. (2008). Historia de la psicología positiva. Antecedentes, aportes $\mathrm{y}$ proyecciones. En M. M. Casullo (Ed.), Prácticas en psicología positiva. Buenos Aires: Lugar.

George, L. K., \& Bearon, L. (1980). Quality of life in older persons. New York, NY: Human Sciences Press.

Gokbayrak, N. S. (2016). Mental health, health behaviors, social support, self-efficacy and disease: An integrative model. (Unpublished doctoral dissertation). University of Rhode Island, Rhode Island, United States. Recuperado de https://goo.gl/3hrT66

González Cabanach, R., Valle Arias, A., Freire Rodríguez, C., \& Ferradás Canedo, M. (2012). Relaciones entre la autoeficacia percibida y el bienestar psicológico en estudiantes universitarios. Revista Mexicana de Psicología, 29, 40-48

Hair, F., Anderson, R. E., Tatham, R. L., \& Black, W. C. (1998). Multivariate data analysis with readings. Upper Saddle River, NJ: Prentice Hall, Inc.

Hayduk, L. A. (1987). Structural Equation Modeling with LISREL, Johns Hopkins University Press, Baltimore, MD.

Hone, L. C., Jarden, A., Schofield, G. M., \& Duncan, S. (2014). Measuring flourishing: The impact of operational definitions on the prevalence of high levels of wellbeing. International Journal of Wellbeing, 4, 62-9. doi:1.5502/ijw.v4i1.4

Huppert, F. A. (2009). A new approach to reducing disorder and improving wellbeing. Perspectives on Psychological Science, 4,108-111. doi:1.1111/j.1745-6924.2009.0110.x

Huppert, F. A., \& So, T. T. (2013). Flourishing across Europe: Application of a new conceptual framework for defining wellbeing. Social Indicators Research, 110(3), 837-861. doi:1.1007/s11205-011-9966-7

Idler, E., \& Benyamini, Y. (1997). Self-rated health and mortality: A review of twentyseven community studies. Journal of Health 
and Social Behavior, 38, 21-37. Recuperado de http://www.jstor.org/stable/2955359

Jackson, L. T., van de Vijver, F. J., \& Fouché, R. (2014). Psychological strengths and subjective well-being in South African white students. Journal of Psychology in Africa, 24(4), 299-307. Recuperado de https://goo.gl/Grh819

Jöreskog, K. G., \& Sörbom, D. (1989). LISREL7: User's reference guide. Mooresville: Scientific Software.

Joshanloo, M., Sirgy, M. J., \& Park, J. (2018). Directionality of the relationship between social well-being and subjective well-being: Evidence from a 20-year longitudinal study. Quality of Life Research, 27, 1-9. doi:10.1007/s11136-018-1865-9

Kern, M., Waters, L., Adler, A., \& White, M. (2015). A multifaceted approach to measuring wellbeing in students: Application of the PERMA framework. Journal of Positive Psychology, 10, 262-271. doi:10.1080/174397 60.2014.936962

Keyes, C. L. (2005). Mental illness and/or mental health? Investigating axioms of the complete state model of health. Journal of Consulting and Clinical Psychology, 73(3), 539-548. doi:1.1037/0022-006X.73.3.539

Keyes, C. L. (2007). Promoting and protecting mental health as flourishing: A complementary strategy for improving national mental health. American Psychologist, 62(2), 95-108. doi:1.1037/0003-066X.62.2.95

Keyes, C. L., \& Simoes, E. J. (2012). To flourish or not: Positive mental health and all-cause mortality. American Journal of Public Health, 102(11), 2164-2172. Recuperado de https://goo.gl/AnAZGN

Keyes, C. L. (1998). Social well-being. Social Psychology Quarterly, 61(2), 121-140.

Klein-Heßling, J., Lohaus, A., \& Ball, J. (2005). Personal risks and resources of health-related behaviour in children. Psychology, Health and Medicine, 10, 31-43.

Kööts-Ausmees, L., \& Realo, A. (2015) The association between life satisfaction and selfreported health status in Europe. European Journal of Personality, 29, 647-657. doi:10.1002/per.2037.
McCullough, M., Emmons, R., Kilpatrick, S., \& Larson, D. (2001). Is gratitude a moral afect?. Psychological Bulletin, 127(2), 249-266. doi:1.1037//0033-2909.127.2.249

McCullough, M, Emmons, R., \& Tsang, J. (2004) Gratitude in intermediate affective terrain: Links of grateful moods to individual differences and daily emotional experience. Journal of Personality and Social Psychology, 86(2), 295-309. doi:1.1037/0022-3514.86.2.295

Lima-Castro, S., Peña-Contreras, E., Cedillo Quizhpe, C., \& Cabrera-Vélez, M. (2017). Adaptación del Perfil PERMA en una muestra ecuatoriana. Eureka (Asunción) en Línea, 14, 69-83. Recuperado de http://psicoeureka.com.py/sites/default/files/ar ticulos/eureka-14-1-12.pdf

Löve, J., Moore, C. D., \& Hensing, G. (2012). Validation of the Swedish translation of the general self-efficacy scale. Quality of Life Research, 21(7), 1249-1253. doi:1.1007/s11136-011-0030-5

Lucas, R. E., Dyrenforth, P. S., \& Diener, E. (2008), Four myths about subjective wellbeing. Social and Personality Psychology Compass, 2, 2001-2015. doi:10.1111/j.1751-9004.2008.00140.x

Luszczynska, A., \& Schwarzer, R. (2005). The role of self-efficacy in health self-regulation. In W. Greve, K. Rothermund, \& D. Wentura (Eds.), The adaptive self: Personal continuity and intentional self-development (pp.137152). Göttingen, Germany: Hogrefe/Huber.

Luszczynska, A., Gutiérrez-Doña, B., \& Schwarzer, R. (2005), General self-efficacy in various domains of human functioning: Evidence from five countries. International Journal of Psychology, 40, 80-89. doi:10.1080/00207590444000041

Lyubomirsky, S., Dickerhoof, R., Boehm, J. K., \& Sheldon, K. M. (2011). Becoming happier takes both a will and a proper way: An experimental longitudinal intervention to boost well-being. Emotion, 11(2), 391. doi:org/1.1037/a0022575

Myers, D. G. (1993) The pursuit of happiness. Discovering the pathways to fulfilment, wellbeing and enduring personal joy, Avon Books, New York 
Olivari-Medina, C., \& Urra-Medina, E. (2007). Autoeficacia y conductas de salud. Ciencia $y$ Enfermería, 13, 9-15. doi:10.4067/S0717-95532007000100002

Park, N., Peterson, C., \& Seligman, M. E. P. (2004). Strengths of character and well-being. Journal of Social and Clinical Psychology, 23(5), 603-619. https://doi.org/10.1521/jscp.23.5.603.50748

Peterson, Ch., \& Seligman, M. (2004) Character Strengths and Virtues. A handbook and classification. New York: APA, Oxford University Press.

Pinquart, M. (2001). Correlates of subjective health in older adults: A meta-analysis. Psychology and Aging, 6(3), 414-26. doi:10.1037//0882-7974.16.3.414

Pressman, S. D., Gallagher, M. W., \& Lopez, S. J. (2013). Is the emotion-health connection a 'first-world problem'? Psychological Science, 24(4), 544-549. doi:10.1177/0956797612457382

Reyes-Jarquín, K., \& Hernández-Pozo, M. (2012). Análisis crítico de los estudios que exploran la autoeficacia y bienestar vinculados al comportamiento saludable. Journal of Behavior, Health \& Social Issues, 3(2), 5-24.

Ryan, M., \& Deci, E. (2001). On happiness and human potentials: A review of research on hedonic and eudaimonic well-being. Annual Review of Psychology, 52,141-166

Schneider, G., Driesch, G., Kruse, A., Wachter, M., Nehen, H. G., \& Heuft, G. (2004). What influences self-perception of health in the elderly? The role of objective health condition, subjective well-being and sense of coherence. Archives of Gerontology and Geriatrics, 39(3), 227-237. doi:1.1016/j.archger.2004.03.005

Schwarzer, R., Bäßler, J., Kwiatek, P., Schröder, K., \& Zhang, J. X. (1997). The assessment of optimistic self-beliefs: Comparison of the German, Spanish, and Chinese versions of the General Self-Efficacy Scale. Applied Psychology, 46, 69-88. doi:1.1111/j.1464-0597.1997.tb01096.x

Seligman, M. (2003). Positive psychology: Fundamental assumptions. American Psychologist, 126-127.
Seligman, M. (2011). Flourish: A visionary new understanding of happiness and well-being. New York, NY: Free Press.

Tsang, J. A. (2006). Brief Report Gratitude and prosocial behaviour: An experimental test of gratitude. Cognition \& Emotion, 20, 138-148. doi:1.1080/02699930500172341

Van Lente, E., Barry, M. M., Molcho, M., Morgan, K., Watson, D., Harrington, J., \& McGee, H. (2012). Measuring population mental health and social well-being. International journal of public health, 57(2), 421-43. doi:1.1007/s00038-011-0317-x

Waure, C. D., Soffiani, V., Virdis, A., Poscia, A., \& Pietro, M. L. D. (2015). Italian university students' self-perceived health and satisfaction of life. Annali dell'Istituto Superiore di Sanità, 51(2), 121-125. doi:1.4415/ANN_15_02_09

Wong, P. T. (2011). Reclaiming positive psychology: A meaning-centered approach to sustainable growth and radical empiricism. Journal of Humanistic Psychology, 51(4). 408-412. doi:1.1177/0022167811408729

Wood, A. M., Froh, J. J., \& Geraghty, A. W. (2010). Gratitude and well-being: A review and theoretical integration. Clinical Psychology Review, 30(7), 890-905. doi:1.1016/j.cpr.201.03.005

Wood, A. M., Maltby, J., Gillett, R., Linley, P. A., \& Joseph, S. (2008). The role of gratitude in the development of social support, stress, and depression: Two longitudinal studies. Journal of Research in Personality, 42(4), 854-871. doi:1.1016/j.jrp.2007.11.003

Wood, A. M., Maltby, J., Stewart, N., Linley, P. A., \& Joseph, S. (2008). A socialcognitive model of trait and state levels of gratitude. Emotion, 8(2), 281. Recuperado de https://goo.gl/nngYGE

World Health Organization. (2004). Promoting mental health: Concepts, emerging evidence, practice: Summary report. Geneva, Switzerland: World Health Organization. Recuperado de https:/goo.gl/saAByH 